Hydroécol. Appl. (2000) Tome 12 Vol. 1-2, pp. 67-86

\title{
Développement des rotifères en Loire moyenne (France)
}

\section{Rotifer growth in the middle Loire river (France)}

\author{
Philippe Gosse \\ EDF, 6 quai Watier 78400 Chatou \\ E-mail : philippe.gosse@edf.fr
}

Résumé. - On présente des mesures de concentration de rotifères effectuées dans l'écoulement avec un pas de temps d'au plus une semaine de mi-juin à mi-septembre 1982 en plusieurs stations de la Loire comprises entre l'amont du confluent de l'Allier et Tours. Un modèle numérique de simulation est mis en œuvre pour reproduire les concentrations mesurées pendant les trois mois. Il apparaît que le taux d'accroissement de la densité du peuplement obtenu par calage du modèle $(0.12$ jour ' en moyenne à $20^{\circ} \mathrm{C}$ en tenant compte de l'effet diluant non négligeable des affluents ou 0.2 jour ${ }^{~}$ à $25^{\circ} \mathrm{C}$ ) est nettement plus faible que les valeurs obtenues expérimentalement in vitro (de l'ordre de 0.5 jour $^{-1}$ à près de $27^{\circ} \mathrm{C}$ ) et que celles habituellement considérées dans la bibliographie dans des milieux également riches en matière nutritive. La discussion de ces résultats porte sur les raisons possibles de cette différence et sur les pistes à creuser pour bien modéliser la dynamique des rotifères en milieu fluvial.

Mots-clés. - plancton, rotifères, eutrophisation, Loire, rivière, modèle, France.

\begin{abstract}
Rotifer concentrations were measured on a weekly basis between mid-June and mid-September 1982 over a $300 \mathrm{~km}$ stretch of the Loire river, from upstream of the Allier confluence down to Tours. A deterministic numerical model was developed to reproduce the concentrations measured in the flow at each station. It appears that the calibrated rate of increase in the population $\left(0.12\right.$ day $^{-1}$ on average at $20^{\circ} \mathrm{C}$ taking into account the significant diluting effect of tributaries, or 0.2 day ${ }^{\prime}$ at $25^{\circ} \mathrm{C}$ ) is much lower than the values found in vitro (close to 0.5 day ' at around $27^{\circ} \mathrm{C}$ ) and considered in literature for similar nutrient-rich water bodies. The reasons for this discrepancy and questions to be examined for a better modelling of rotifer dynamics in rivers are discussed.
\end{abstract}

Keywords. - plankton, rotifers, eutrophication, Loire, river, model, France. 


\section{INTRODUCTION}

Dans le but d'améliorer un modèle numérique d'eutrophisation mis au point sur la Loire moyenne entre Cosne et le confluent de l'Indre (Gosse, 1981), une campagne spécifique de mesures de qualité d'eau a été effectuée en 1982 entre le 14 juin et le 16 septembre, période à plus fort développement planctonique: 11 stations comprises entre l'amont du confluent de l'Allier et l'aval de Saumur ont été visitées deux fois par semaine durant cette période, le suivi se déroulant à chaque fois sur deux jours, les 6 stations les plus aval étant visitées un jour après les stations amont (voir figure 1 pour l'emplacement des stations).

Dans le cadre de cette campagne, des comptages de zooplancton ont été effectués. Plus lourds à mettre en $œ u v r e$ que le suivi des autres paramètres étudiés en routine (notamment chlorophylle, phosphore, azote et silice), ils n'ont été effectués qu'en 5 stations, et seulement au milieu de l'écoulement principal (les autres paramètres étant en outre analysés en deux autres points de la transversale) : une sur l'Allier au site de Le Guétin situé à l'amont immédiat du confluent de la Loire, et quatre sur la Loire à

- Imphy dans le proche amont du confluent de l'Allier,

- Sully, près de $120 \mathrm{~km}$ en aval du confluent,

- Muides, près de $90 \mathrm{~km}$ en aval de Sully,
- et Tours, près de $75 \mathrm{~km}$ en aval de Muides.

Compte tenu de l'importance pour le calage d'un modèle fluvial d'eutrophisation, de la mise à disposition d'une chronique suffisamment fine des conditions limites amont, les rotifères - identifiés comme le principal groupe consommateur du phytoplancton dans le fleuve (Champ,1980; Pourriot et Champ, 1982) - ont été comptés sur tous les prélèvements d'eau effectués sur l'Allier et la Loire amont. On dispose ainsi d'une chronique bi-hebdomadaire de mesures de densité de rotifères aux deux stations amont. Le comptage aux autres stations a été réalisé sur une base hebdomadaire.

En complément de ces données in situ, quelques échantillons d'eau ont été prélevés en Loire dans le proche amont de la station de Muides - au droit de la centrale de Saint Laurent des Eaux - et ont été mis à incuber in vitro pour déterminer expérimentalement le taux d'accroissement des rotifères dans des conditions artificielles d'éclairement reproduisant le cycle jour-nuit de lumière.

Bien qu'elles soient anciennes, il semble utile de faire connaître ces données de zooplancton de 1982 qui n'ont pas fait l'objet de publications jusqu'ici - pour au moins trois raisons :

- c'est la première année pour laquelle on peut déterminer sur un été et sur une longue distance un taux d'accroissement du peuplement de rotifères en Loire 


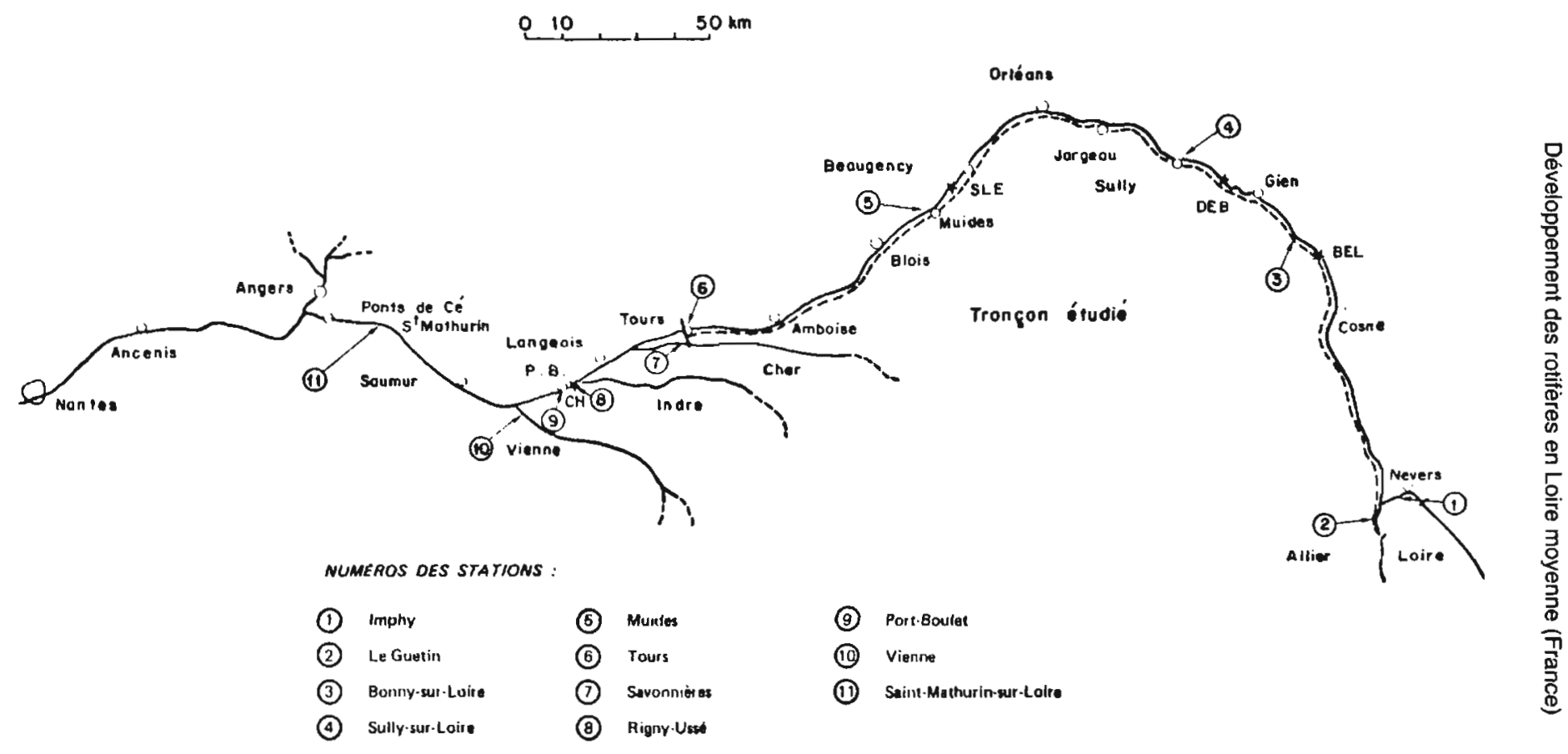

- BEL, OEB, SLE, CH : Cantralos the Belleville, Oempierreen Burly, Saint-Laurent, Chinon.

Fig. 1 - Tronçon de Loire étudie

Fig. 1 - Stretch studied on the Loire river 
moyenne à partir de mesures in situ. Les principaux travaux au cours des années précédentes Champ, 1977; Lair et Sargos, 1981; Pourriot et Champ, 1982; Pourriot et al, 1982 - ne permettent pas véritablement un tel calcul, faute d'une fréquence de mesures adaptée pour suivre les mêmes masses d'eau entre stations.

- il y a eu relativement peu d'études conduites sur les rotifères de la Loire moyenne les années suivantes (Lair et al. 1997, 1998, 1999, 2000).

- l'exploitation de ces données permet d'apporter des éléments de réflexion sur des questions non résolues sur la dynamique des rotifères en Loire moyenne, et aussi de soulever le problème de la représentativité, d'une part d'expérimentations in vitro, et d'autres part de modèles numériques de simulation de rotifères développés sur d'autres rivières.

\section{PROTOCOLE DE MESURES}

\subsection{Prélèvement et comptage du zooplancton}

En chaque station, dans le but d'évaluer la densité des rotifères dans l'écoulement fluvial, 50 litres d'eau ont été prélevés à chaque visite au seau à partir d'un pont, en se plaçant au milieu de l'écoulement. Les prélèvements successifs pour obtenir le volume d'eau désiré ainsi que la faible profondeur et la vitesse moyenne relativement élevée de la Loire moyenne limitent les erreurs possibles de représentativité de l'échantillon d'eau collecté. Les 50 litres d'eau récupérés ont été filtrés sur place sur une soie à bluter de $40 \mathrm{mi}$ cromètres de maille, la fraction filtrée étant recueillie dans des bocaux en polyéthylène de $500 \mathrm{ml}$ avec ajout de formol à $5 \%$ en volume pour la conservation.

Dans les 6 mois après prélèvement in situ, $3 \mathrm{ml}$ de la suspension homogénéisée de zooplancton sont extraits des bocaux et examinés sous loupe binoculaire dans une cuvette de Dolfuss de $11 \times 6 \mathrm{~cm}$ à fond quadrillé, les zooplanctontes trouvés étant comptés.

Ont ainsi été comptés: les rotifères (sans détermination spécifique), les cladocères et les copépodes, ces derniers étant présents uniquement sous forme larvaire (nauplii et copépodites) ; il n'y avait pas de zooplancton carnivore dans les échantillons, mis à part des gros rotiferes inclus dans le comptage des rotifères, comme Asplanchna qui représente une très faible proportion des rotifères en Loire moyenne sur les données disponibles pour les années antérieures à 1998 (Lair et al. 1997, Lair et al. 1998, Lair et al. 1999).

On reconstitue la densité $\mathrm{N}$ d'individus par litre dans la rivière à partir de la densité $n$ trouvée dans la cuvette par l'équation $N=(n V d) /(v F)$, avec $F$ volume filtré initial $(50 \mathrm{I}), \mathrm{V}$ le volume du filtrat (variant de 300 à $500 \mathrm{ml}$ ), $v$ le volume de comptage 
$(3 \mathrm{ml})$ et d facteur de dilution du filtrat de $3 \mathrm{ml}$ (la dilution apparaissant nécessaire dans les cas de très fortes densités).

\subsection{Prélèvements et incubations in vitro}

A plusieurs occasions au cours de l'été 1982 , de l'eau de Loire a été prélevée au droit du canal de la centrale de Saint Laurent B, en absence d'échauffement, la centrale étant à l'arrêt pendant toute la période des prélèvements.

Aussitôt transportée, en moins de 3 heures, à température ambiante jusqu'au laboratoire EDF de Chatou, l'eau est mise à incuber sans filtration préalable.

L'incubation est réalisée avec un appareil de culture ayant les caractéristiques suivantes:

- plateau de $0.21 \mathrm{~m}^{2}$ rotatif de manière à permettre une agitation des flacons, la vitesse de rotation étant réglée afin d'éviter la sédimentation des algues planctoniques

- flacons contenant un litre d'échantillon et recouverts d'un film translucide

- éclairage commandé par une horloge comprenant quatre tubes "lumière du jour" de $20 \mathrm{~W}$ délivrant un éclairement de $60 \mathrm{~W} / \mathrm{m}^{2}$ entre 400 et $700 \mathrm{~nm}$. Le cycle jour/nuit a été reproduit avec une durée d'éclairement de 10 heures ou 8 heures par jour
- pas de régulation thermique, la température relevée régulièrement oscillant entre 26 et $29^{\circ} \mathrm{C}$.

On s'intéresse ici aux incubations au cours desquelles des comptages de rotifères - selon la méthode précisée au chapitre 2.1 - ont été réalisés; elles correspondent à des prélèvements d'eau effectués en Loire à trois reprises en milieu de journée: le 26 juillet (température d'eau au lieu de prélèvement de $\left.26^{\circ} \mathrm{C}\right)$, le $1^{\text {er }}$ septembre $\left(18^{\circ} \mathrm{C}\right)$ et le 13 septembre $1982\left(23^{\circ} \mathrm{C}\right)$.

\section{PRÉSENTATION DES RÉSULTATS}

\subsection{Tendance d'évolution amont- aval donnée par les mesures in situ}

On constate tout d'abord (fig. 2) que la Loire amont était beaucoup plus riche en rotifères que l'Allier au cours de l'été 1982, la densité moyenne sur le fleuve amont $\left(0.46^{\star} 10^{6}\right.$ individus $\left./ \mathrm{m}^{3}\right)$ étant de l'ordre du double de son affluent $\left(0.2^{*} 10^{6}\right.$ individus $\left./ \mathrm{m}^{3}\right)$. Ce rapport 2 se retrouve en flux (fig. 3 ), les débits de la Loire amont et de l'Allier étant tous deux voisins de $40 \mathrm{~m}^{3} / \mathrm{s}$ en moyenne sur les jours de mesure ( $43 \mathrm{~m}^{3} / \mathrm{s}$ et $38 \mathrm{~m}^{3} / \mathrm{s}$ respectivement). Si bien que l'effet de l'Allier était de diminuer de l'ordre du quart les concentrations de rotifères de la Loire en moyenne sur l'été. 


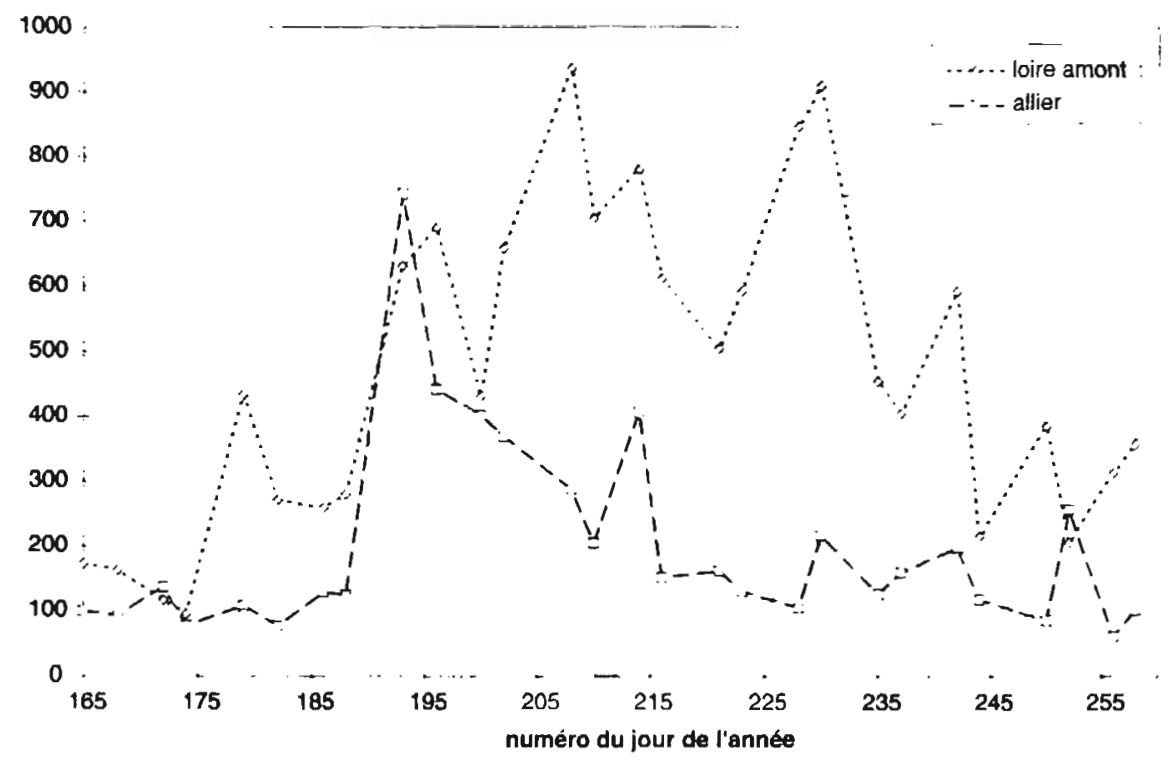

Fig. 2 - Concentration de rotifères au confluent de la Loire et de l'Allier en 1982 (individus / litre) Fig. 2 - Rotifer concentration at the confluent of the Loire and the Allier river in 1982 (individuals/itre)

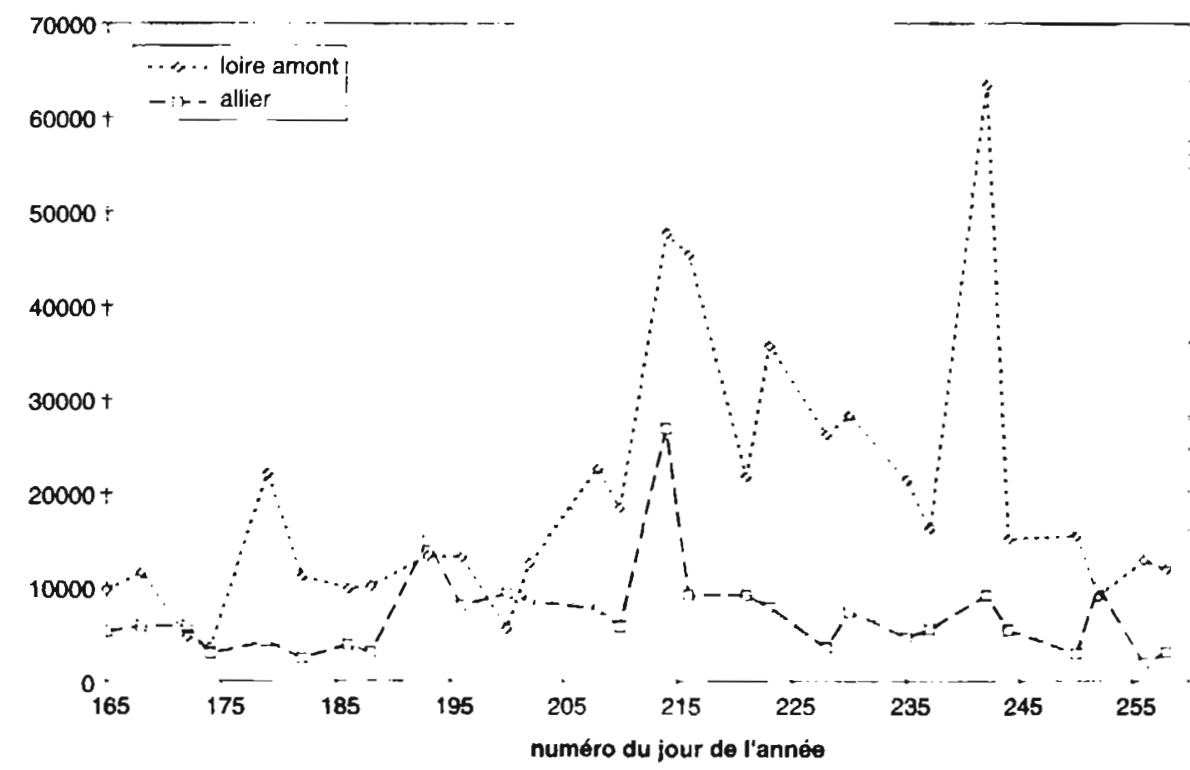

Fig. 3 - Flux de rotifères au confluent de la Loire et de l'Allier en 1982 (milliers d'individus / seconde)

Fig. 3 - Rotifer flux at the confluent of the Loire and the Allier in 1982 (individuals $\times 1,000 /$ litre) 
En reconstituant une densité de mélange à partir des mesures de Imphy et du Guetin et des débits moyens journaliers aux stations hydrologiques voisines, on dispose ainsi de 4 points de Loire permettant de suivre l'évolution des densités de rotifères dans le fleuve sur près de 300 kilomètres, depuis le confluent de l'Allier jusqu'à Tours.

On s'aperçoit (figs. 4 et 5) qu'il n'y a pas d'augmentation visible de la concentration de rotifères d'amont en aval, sauf faiblement, après le 10 août (jour 222). Le fait est que la concentration moyenne des mesures effectués est de $0.41^{*} 10^{6}$ individus $/ \mathrm{m}^{3}$ à Sully et à Muides et de $0.49^{*} 10^{6}$ individus $/ \mathrm{m}^{3}$ à Tours. A relativiser compte tenu de l'hétérogénéité des masses d'eau et l'incertitude de mesure, une légère diminution - potentiellement favorisée par les apports de débit dans le proche aval d'Orléans (voir plus loin au chapitre 3.2) - apparaît dans la deuxième quinzaine de juillet entre la station de mélange Loire amont-Allier et celles intermédiaires de Sully et Muides, mais elle disparaît si l'on compare la station amont à la station la plus aval (fig. 5).

A plus petite échelle spatiale, les campagnes de mesures bimensuelles de Lair et collaborateurs (Lair et al. 1997, Lair et al. 1998, Lair et al. 1999) effectuées depuis 1995 aux stations intermédiaires de Dampierre (près de Sully) et Saint Laurent des Eaux (près de Muides) distantes de $100 \mathrm{~km}$, ne mettent pas non plus en évidence d'augmentation significa- tive de la densité du peuplement de rotifères entre les 2 stations : sur l'ensemble des 3 années 1995-1997, la moyenne de concentration sur la période juin-octobre apparaît de $1.2^{\star} 10^{6}$ individus $/ \mathrm{m}^{3}$ à la station amont de Dampierre et de $1.0^{*} 10^{6}$ individus $/ \mathrm{m}^{3}$ à la station aval de Saint Laurent. On remarque que c'est un niveau moyen à peu près deux fois supérieur à celui trouvé durant notre campagne entre mi-juin et mi-septembre 1982 (un peu plus de $0.4{ }^{\star} 10^{6}$ individus $/ \mathrm{m}^{3}$ ).

Les comptages de zooplancton de la campagne de 1982 confirment par ailleurs la faible présence de cladocères, copépodites et nauplii, qui avaient une concentration de respectivement $3^{\star} 10^{3}$ individus $/ \mathrm{m}^{3}$, $3^{\star} 10^{3}$ individus $/ \mathrm{m}^{3}$ et $5^{\star} 10^{3}$ indivi$\mathrm{dus} / \mathrm{m}^{3}$, en moyenne au cours de l'été pour l'ensemble des trois stations de Sully, Muides et Tours.

\subsection{Détermination par modélisation de la vitesse d'accroissement du peuplement des rotifères}

Il s'agit ici d'aller un peu plus loin que la simple analyse visuelle de la figure 4 et d'essayer de préciser la valeur du taux d'accroissement de la densité des rotifères sur la période d'étude en tenant compte des conditions hydrauliques et thermiques de l'écoulement.

On utilise pour cela un modèle numérique déterministe qui reprend la structure hydrodynamique du modèle non permanent d'eutrophisation de la 


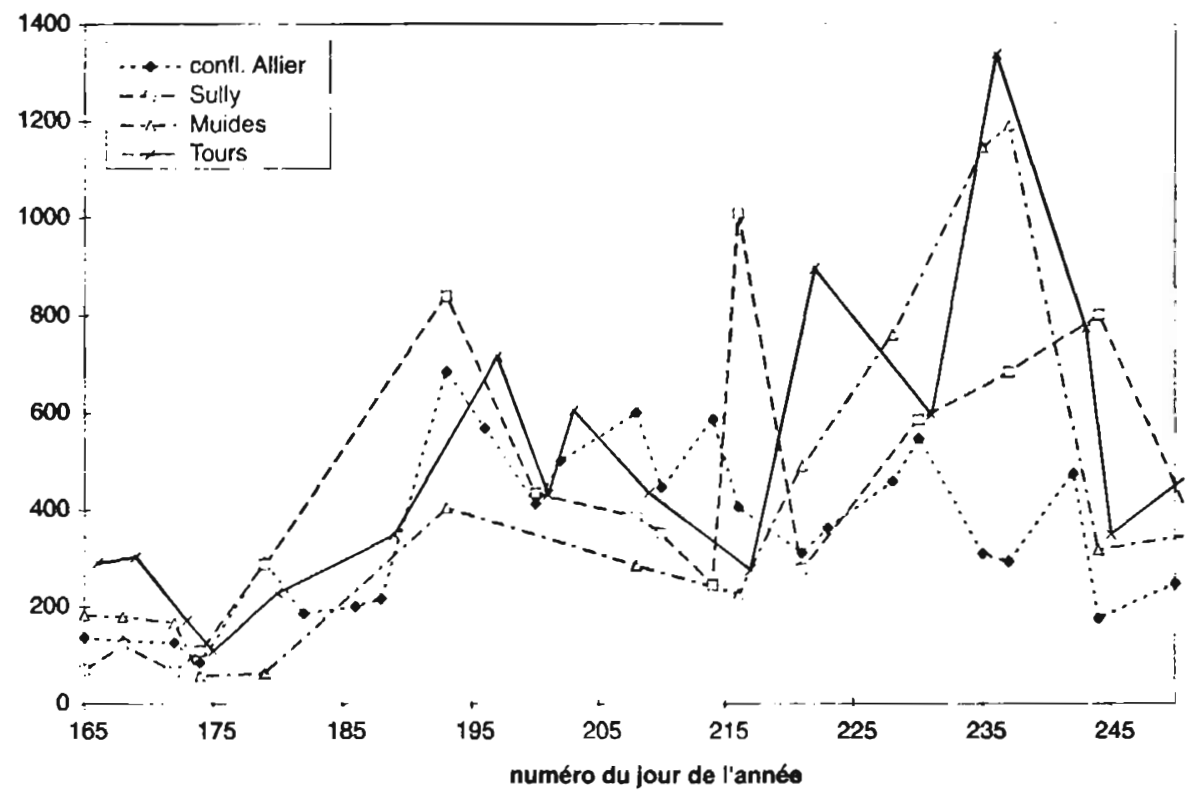

Fig. 4 - Concentration de rotifères en quatre stations de la Loire moyenne en 1982 (individus / litre) Fig. 4 - Rotifer concentration at 4 sites on the middle Loire in 1982 (individuals/litre)

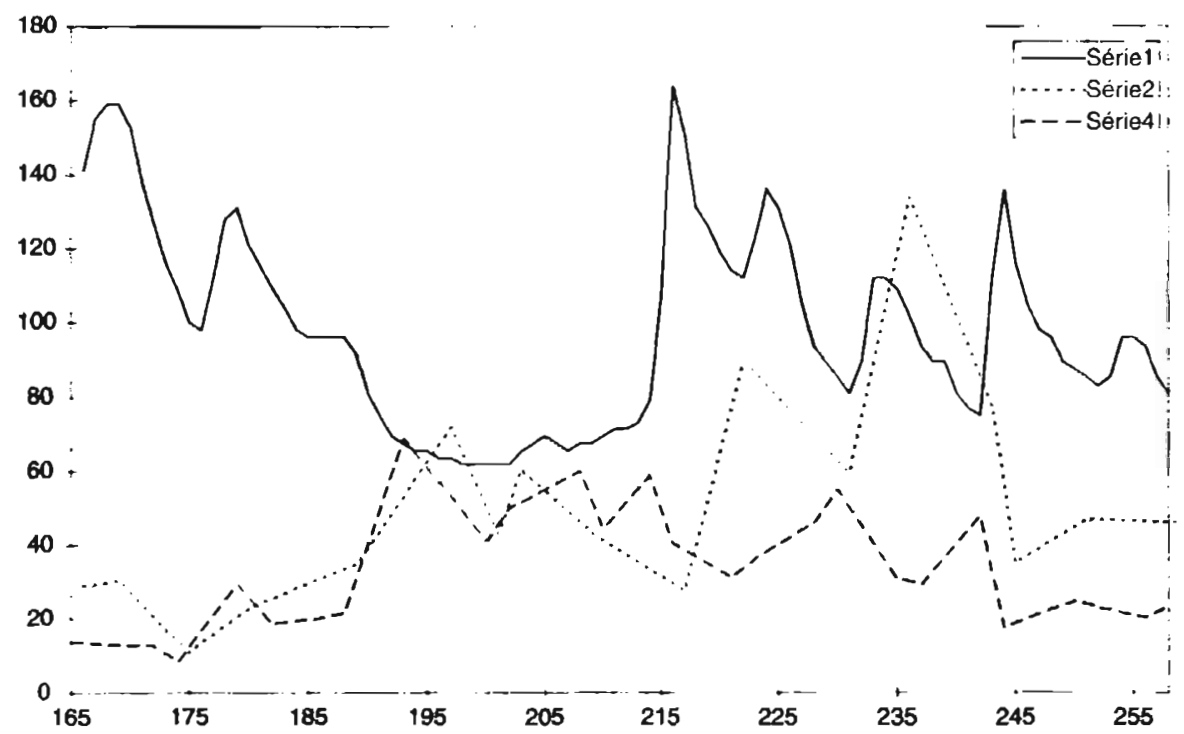

Fig. 5 - Débits moyens journaliers en $\mathrm{m} 3 / \mathrm{s}$ de la Loire a Gien (trait continu) et teneurs en rotifères en Loire au confluent de l'Allier (trait discontinu) et à Tours (pointillé) en individus/décilitre durant l'été 1982

Fig. 5 - Mean daily discharge in $\mathrm{m} 3 / \mathrm{s}$ in the Loire in Gien (continuous line) and rotifer concentrations at the confluence with the Allier (broken line) and at Tours (dotted line), in individuals/decilitre. summer 1982. 
Loire développé en 1981 (Gosse, 1981) en déplaçant sa limite amont jusqu'au confluent de l'Allier. Le modèle a ici pour principale variable la densité de rotifères transportée par l'écoulement, soit la variable $Z$ exprimée en individus par $\mathrm{m}^{3}$.

L'équation de bilan de $\mathrm{Z}$ résolue à pas horaire est la suivante :

$$
\mathrm{dZ} / \mathrm{dt}=\mathrm{A} 20^{\star} \mathrm{f}(\mathrm{T})
$$

avec A20 (en jour ${ }^{-1}$ ) vitesse d'accroissement de $\mathrm{Z}$ à $20^{\circ} \mathrm{C}$, qui constitue un paramètre de calage, $T$ température $\left({ }^{\circ} \mathrm{C}\right.$ ) de la rivière (qui varie entre $18^{\circ} \mathrm{C}$ et $27^{\circ} \mathrm{C}$ en moyenne journalière sur la période d'étude) et $f$ fonction traduisant l'évolution du taux d'accroissement en fonction de la température.

Dans leur modèle récent de rotifères développé sur la Meuse, Everbecq et al. (2000) proposent pour $\mathrm{f}$, l'équation $\mathrm{f}(\mathrm{T})=\mathrm{e}^{\mathrm{TT} 2 \mathrm{~T}_{\mathrm{i}+10}}$ ce qui donne à $25{ }^{\circ} \mathrm{C}$ un taux d'accroissement plus élevé de $65 \%$ à celui de $20^{\circ} \mathrm{C}$.

On reprend cette formulation qui donne des résultats assez proches de celle retenue par Allan (1976) pour le taux de croissance des rotifères. Allan propose en effet $f=0.165^{\star} e^{0.99} r$, ce qui donne un taux d'accroissement à $25^{\circ} \mathrm{C}$ plus élevé de $55 \%$ à celui de $20^{\circ} \mathrm{C}$.

On considérera toutefois, qu'au dessus de $25^{\circ} \mathrm{C}$, on a $\mathrm{f}(\mathrm{T})=\mathrm{f}\left(25^{\circ} \mathrm{C}\right)$, sur la base des travaux de Pourriot et al. (1982), qui situent l'optimum de croissance vers $25^{\circ} \mathrm{C}$ en Loire moyenne, même si certaines espèces thermophiles peuvent dans certaines conditions représenter une proportion non négligeable du peuplement, comme Sinantherina socialis, dont l'optimum de croissance est proche de $28^{\circ} \mathrm{C}$ (Champ, 1977 et 1980).

Dans le modèle développé ici, seules les mesures de rotifères disponibles aux deux stations amont Imphy et Le Guétin sont nécessaires pour le calcul proprement dit. Elles servent à reconstituer les valeurs journalières de mélange de $Z$ au confluent Loire amont-Allier, c'est à dire aux conditions limites amont du modèle. Le calage du modèle consiste à régler A20 en sorte de minimiser - visuellement et à l'aide de la méthode des moindres carrés - les écarts calcul-mesures aux trois stations de Loire situées plus en aval.

Comme pour le modèle établi en 1981 , la température de l'eau est calculée à pas de temps horaire et l'évolution temporelle et longitudinale des débits journaliers est constituée à partir des enregistrements disponibles à des stations hydrologiques. Dans cette étude, on considere les stations suivantes (données accessibles dans la banque française HYDRO) : Le Guetin sur l'Allier, et Nevers (dans le proche amont du confluent de l'Allier), Gien, Orléans et Blois sur la Loire.

Sur la période analysée allant de mi-juin à mi-septembre, le débit moyen de la Loire est de $79 \mathrm{~m} / \mathrm{s}$ aux conditions limites amont (en sommant les débits aux stations Nevers et Le Guetin), $98 \mathrm{~m}^{3} / \mathrm{s}$ à Gien (voir profil sur la figure 5 ), $83 \mathrm{~m}^{3} / \mathrm{s}$ à Orléans et $106 \mathrm{~m}^{3} / \mathrm{s}$ à Blois. Des ap- 
ports d'eau conséquents apparaissent donc, d'une part entre les 2 points amont de mesures de rotifères et la station de Gien, et d'autre part entre Orléans et Blois.

Le “ trou » de débit à Orléans s'explique pour une bonne part par l'infiltration de la Loire en amont d'Orléans et l'apport de la nappe (Beauce + résurgence Loire), du Loiret et des rivières de Beauce dans le proche aval de la ville. Il est à noter qu'une étude de R. Gonzalez (1991) a réévalué à près de $12 \mathrm{~m}^{3} / \mathrm{s}$ les résurgences de nappe en été (1985-1986) dans le proche aval d'Orléans dans le lit même de la Loire, soit un niveau à peu près comparable à celui précédemment proposé $\left(10 \mathrm{~m}^{3} / \mathrm{s}\right)$ par Crouzet et Receveur (1977).

Nous considérerons que ces deux apports (pris égaux respectivement à $19 \mathrm{~m}^{3} / \mathrm{s}$ et $23 \mathrm{~m} / \mathrm{s}$ ) sont dépourvus de zooplancton, ce qui s'impose pour des apports de nappe et peut se justifier pour des apports de petits cours d'eau. Cela permet de ne pas sousestimer le taux d'accroissement du peuplement de rotifères, une erreur relevée dans la bibliographie par Viroux (2000) : elle consiste à analyser des concentrations d'organismes entre 2 points fluviaux sans tenir compte des apports de débit intermédiaires. Nous verrons plus loin la différence obtenue pour le taux d'accroissement du peuplement en supposant que ces apports ne changent pas la densité de rotifères de la Loire à leur point de rejet dans le fleuve.

Les fonctions vitesse $=f(Q)$ et profondeur $=f(Q)$ retenues dans le mo- dèle sont celles réactualisées en 2000 par EDF pour la mise au point d'un modèle radioécologique de la Loire (Siclet et al., article à paraître). Ainsi, pour un débit moyen de $100 \mathrm{~m}^{3} / \mathrm{s}$, il faut un peu plus de 7 jours aux masses d'eau pour parcourir le tronçon ligérien modélisé bec d'AllierTours.

II apparaît que le réglage suivant de A20 permet au modèle de reproduire assez convenablement les teneurs en rotifères observées aux 3 stations aval (fig. 6) :

- A20 = 0.1 jour $^{-1}$ du 14 juin au 8 juillet (jours 165 à 189) et 0.05 jour $^{-1}$ du 9 juillet au 9 août (jours 190 à 221), la température moyenne journalière étant voisine de $20^{\circ} \mathrm{C}$ des jours 165 à 180, augmentant progressivement par la suite jusqu'à $27^{\circ} \mathrm{C}$ niveau atteint mi-juillet, puis diminuant progressivement jusqu'à $22-23^{\circ} \mathrm{C}$, niveau atteint le 2 août et se maintenant jusqu'au 12 août

- A20 $=0.22$ jour $^{-1}$ du 10 août au 27 août (jours 222 à 239), la température moyenne journalière baissant progressivement de 22 "C à $18^{\circ} \mathrm{C}$ du 13 août au 27 août

- A20 = 0.15 jour $^{-1}$ du 28 août au 16 septembre (jours 241 à 259), la température moyenne journalière augmentant progressivement de 18 à $23^{\circ} \mathrm{C}$ dans l'intervalle

Une autre application du modèle permet d'établir que les valeurs calées de A20 seraient inférieures de 0.05 jour: aux valeurs indiquées cidessus si l'on avait considéré que les 

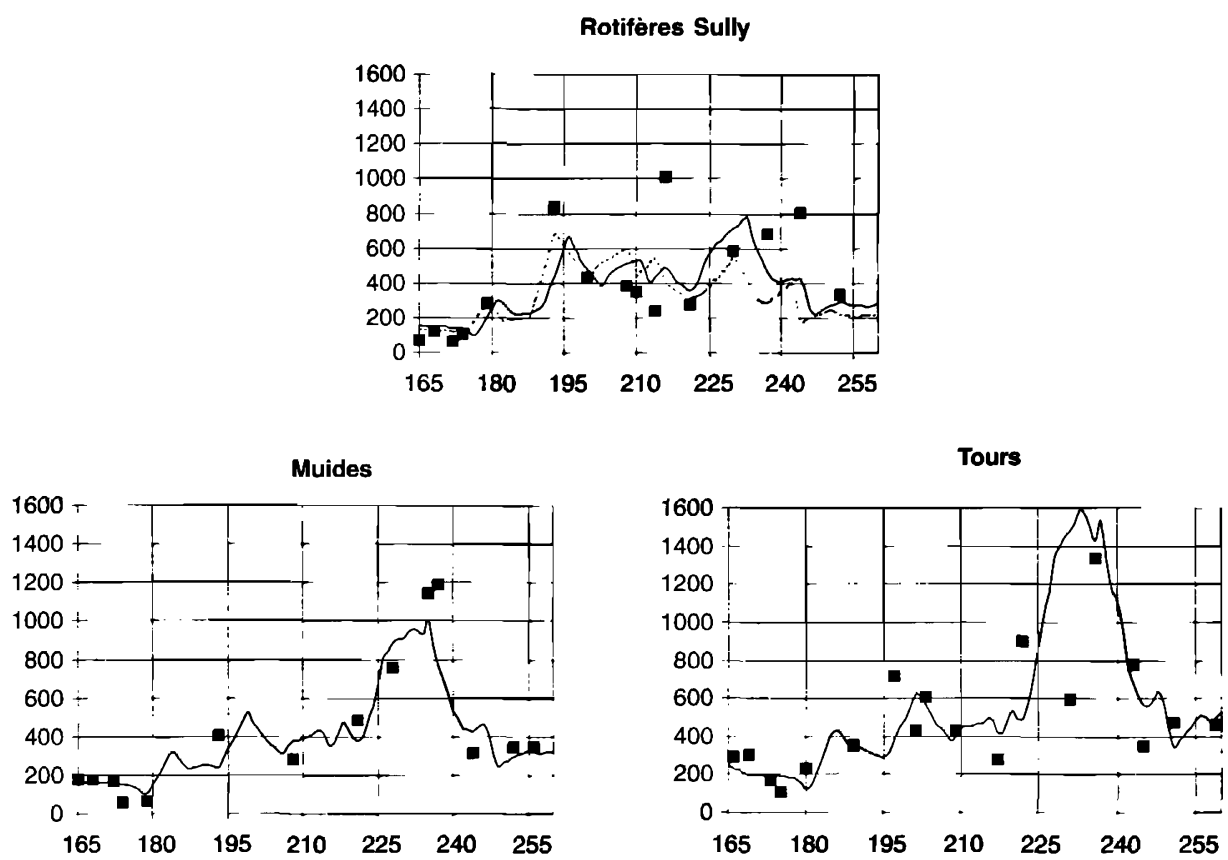

Fig. 6 - Comparaison calcul-mesures pour les densités de rotifères en Loire (en individus/litre) avec un réglage optimisé de A20 sur l'ensemble de l'été 1982 à partir du confluent de l'Allier. La courbe en pointillé sur la Figure de Sully est la chronique d'entrée du modèle au confluent de l'Allier.

Fig. 6 - Comparison for summer 1982 between calculations and measurements of rotifer densities in the Loire (in individuals/litre), with an optimized setting for A20 downstream of the confluent with the Allier. The dotted curve for the Sully station is the input profile for the model at the confluence with the Allier.

apports de débit entre l'aval du confluent de l'Allier et Tours ne modifiaient pas la concentration de rotifères de la Loire à leur point de rejet dans le fleuve.

Comme il est probable que les apports de débit en aval du confluent de l'Allier ne sont pas tous dépourvus de rotifères - sans pour autant atteindre a priori les niveaux dans le fleuve-, il apparaît possible que l'hypothèse retenue d'absence de rotifères dans ces apports puisse conduire à une surestimation de A20 de l'ordre de $0.01-0.02$ jour $^{-1}$.

En conclusion, on retrouve bien par le modèle une tendance d'accroissement plus élevée du peuplement de rotifères après le 10 Août, tel que suggéré par le premier examen des figures 4 et 5 . Mais, on se rend compte que le simple examen de cette figure - qui ne permet pas d'intégrer l'effet des variations longitudinales de débit - tend à minimiser le taux général d'accroissement. 
II est important de noter que A20 aurait été encore plus faible et en moyenne très proche de zéro si en plus de celui des apports aval, on avait également négligé l'effet diluant conséquent de l'Allier. On rappelle en effet que le niveau moyen de densités de rotifères au cours de l'été donné par les mesures en Loire à Tours $\left(0.49^{\star} 10^{6}\right.$ individus $\left./ \mathrm{m}^{3}\right)$ est très proche de celui trouvé sur le fleuve à Imphy dans le proche amont du confluent de l'Allier $\left(0.46^{*} 10^{6}\right.$ individus $\left./ \mathrm{m}^{3}\right)$.

\subsection{Comparaison avec la détermination in vitro du taux d'accroissement des rotifères}

Faite sur une eau brute non enrichie en nutriments, l'incubation sur le prélèvement d'eau de Saint Laurent du 13 septembre révèle une augmentation moyenne de la densité de rotifères de $0.35^{\star} 10^{6}$ individus $/ \mathrm{m}^{3}$ à $9.3^{*} 10^{6}$ individus $/ \mathrm{m}^{3}$ en 172 heures (du $13 / 9$ au 20/9), ce qui donne une vitesse d'accroissement du peuplement de 0.46 jour '; en fin d'incubation, les rotiferes étaient plutôt de grande taille avec des Brachionus calyciflorus dominants, alors qu'ils étaient plutôt de petite taille, plus diversifiés avec des Keratella relativement abondants au début de l'incubation.

Faites sur une eau brute enrichie en nutriments :

- l'incubation réalisée à partir du prélèvement du 26 juillet (teneurs en P-PO, passant de 0.015 a $0.18 \mathrm{mg} / \mathrm{l}$, de $\mathrm{N}-\mathrm{NO}_{3}$ de 0.23 à
$1.9 \mathrm{mg} / \mathrm{l}$, pour une teneur en $\mathrm{SiO}_{2}$ non modifiée de $6.5 \mathrm{mg} / \mathrm{l})$ montre une augmentation de la densité de rotifères de $0.07^{*} 10^{6}$ individus $/ \mathrm{m}^{3}$ à $4^{*} 10^{6}$ individus $/ \mathrm{m}^{3}$ en 99 heures (du 26/7 au 30/7), ce qui donne un taux d'accroissement du peuplement de 0.66 jour' ${ }^{\prime}$. Au cours de l'incubation la teneur en chlorophylle a corrigée - indicateur de biomasse phytoplanctonique (Khalanski et al., 1981) - croît de $170 \mathrm{mg} / \mathrm{m}^{3}$ à $470 \mathrm{mg} / \mathrm{m}^{3}$, valeur atteinte au bout de 75 heures puis retombe à $120 \mathrm{mg} / \mathrm{m}^{3}, 24$ heures après. A titre indicatif, sur l'eau brute non enrichie en nutriments sur laquelle les rotifères n'ont pas été comptés en cours d'incubation - , la teneur en chlorophylle a corrigée croît de $170 \mathrm{mg} / \mathrm{m}^{3}$ à $270 \mathrm{mg} / \mathrm{m}^{3}$, valeur atteinte $27 \mathrm{heu}$ res $(27 / 7)$ et 51 heures $(28 / 7)$ après le début de l'incubation, décroît lentement jusqu'à $220 \mathrm{mg}$ chla/m sur les 24 heures suivantes (29/7), avant de chuter à $95 \mathrm{mg}$ chla $/ \mathrm{m}^{3}$ sur les dernières 24 heures $(30 / 7)$, un niveau à peine inférieur à celui trouvé dans l'échantillon enrichi en nutriments.

- l'incubation réalisée à partir du prélèvement du $1^{\text {" }}$ septembre (teneurs en $\mathrm{P}-\mathrm{PO}_{4}$ passant 20 heures après le prélèvement de 0.009 à $0.6 \mathrm{mg} / \mathrm{l}$, de $\mathrm{N}-\mathrm{NO}_{3}$ de 0.18 à $2.2 \mathrm{mg} / \mathrm{l}$, et de $\mathrm{SiO}$, de $3.5 \mathrm{mg} / \mathrm{là}$ $30 \mathrm{mg} / \mathrm{l}$ ) donne un accroissement moyen de la densité de rotifères de $0.405^{*} 10^{6}$ individus $/ \mathrm{m}^{3}$ à $15.7^{*} 10^{6}$ individus $/ \mathrm{m}^{3}$ en 146 heures (du $1 / 9$ au $7 / 9$ ), ce qui correspond à un 
taux de croissance de 0.60 jour $^{-1}$, les rotifères en fin d'incubation apparaissant tous vivants et dominés par Brachionus calyciflorus (dont de nombreux individus ovigères) associés à quelques Keratella. Au cours de l'incubation la teneur en chlorophylle a corrigée a été mesurée à $170 \mathrm{mg} / \mathrm{m}^{3}$ le 2/9, 20 heures après le prélèvement, à $245 \mathrm{mg}$ $/ \mathrm{m}^{3}$ le $3 / 9,44$ heures après le prélèvement, de $70 \mathrm{mg} / \mathrm{m}^{3}$ le $6 / 9$, 116 heures après le prélèvement, de $35 \mathrm{mg} / \mathrm{m}^{3}$ les $7 / 9$ et $8 / 9$, respectivement 146 et 164 heures après le prélèvement. A titre indicatif, sur l'eau brute non enrichie sur laquelle les rotifères n'ont pas été comptés en cours d'incubation, la teneur en chlorophylle a corrigée a été mesurée à $170 \mathrm{mg} \mathrm{chla} / \mathrm{m}^{3}$ le $2 / 9,20$ heures après le prélèvement, à $245 \mathrm{mg} \mathrm{chla} / \mathrm{m}^{3}$ le $3 / 9$, 44 heures après le prélèvement, de $50 \mathrm{mg} / \mathrm{m}^{3}$ le $6 / 9,116$ heures après le prélèvement et de $85 \mathrm{mg} / \mathrm{m}^{3}$ le $8 / 9,164$ heures après le prélèvement.

On constate donc que le taux d'accroissement du peuplement de rotifères trouvé in vitro est nettement plus élevé que celui donné par l'exploitation des mesures de densité de rotifères en rivière : le modèle (chapitre 3.2) donne en effet un taux d'accroissement à $20^{\circ} \mathrm{C}$ de 0.05 jour $^{-1}$ fin juillet et 0.15 jour $^{-1}$ en septembre, soit respectivement 0.08 jour ${ }^{-1}$ et 0.25 jour $^{-1}$ à $27^{\circ} \mathrm{C}$, température moyenne des incubations. Cela représente un écart d'accroissement d'au moins 0.2 jour $^{-1}$.

\subsection{Comparaison avec des données de la bibliographie}

S'ils apparaissent inférieurs à ceux que donne le modèle de la Loire présenté au chapitre 3.2, les niveaux de taux d'accroissement trouvés avec les incubations in vitro sur de l'eau prélevée à Saint Laurent sont dans la gamme des taux de croissance donnés par Allan (1976) pour les rotifères (0.2 à 1.5 jour $\left.^{-1}\right)$.

On sait par ailleurs que des taux de croissance particulièrement élevés peuvent être obtenus en élevage de masse, par exemple pour Brachionus plicatilis utilisé pour nourrir des larves de poissons et crustacés, avec un taux de croissance de 1.1 jour ' à $15^{\circ} \mathrm{C}$ (Liu Fennggi, 1996).

S'appuyant sur l'analyse bibliographique fouillée et expérimentale de Gosselain (1998) sur la filtration des rotifères en milieu fluvial, et sur les travaux de Viroux (2000) sur la dynamique du métazooplancton mosan, Everbecq et al. (2000) proposent un modèle déterministe fluvial de rotifères (décomposés en rotifères de type Brachionus et en rotifères de type $\mathrm{Ke}$ ratella) qui conduit dans l'application faite pour la Meuse, à un taux d'accroissement du peuplement dépassant 0.3 jour ' à $20^{\circ} \mathrm{C}$ à l'échelle de la centaine de kilomètres, près de 400 kilomètres en aval de la source du fleuve.

En effet Everbecq et al. retiennent pour les rotifères: un taux moyen de filtration de 1.5 litre d'eau/mgC de rotifère/jour à $20^{\circ} \mathrm{C}$ et la transformation en biomasse zooplanctonique de 

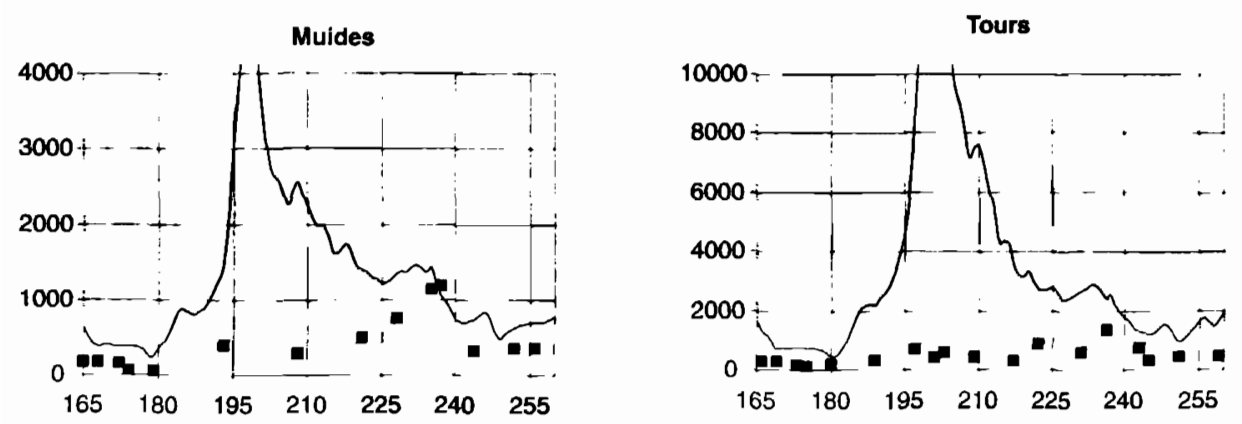

Fig. 7 - Comparaison calcul- mesures pour les densités de rotifères (en individus par litre) aux stations de Muides et Tours, en prenant A20 $=0.3$ jour 'sur l'ensemble de l'été 1982 à partir du confluent de l'Allier.

Fig. 7 - Comparison for summer 1982 between calculations and measurements of rotifer densities (in individuals/litre) at the stations of Muides and Tours, taking A20 $=0.3$ days downstream of the confluence with the Allier.

$12 \%$ du phytoplancton comestible filtré. Comme le phytoplancton comestible atteint $4 \mathrm{mgC/l}$ (soit près de $100 \mathrm{mg} \mathrm{chla} / \mathrm{m}^{3}$, niveau toujours dépassé en Loire moyenne durant l'été 1982) sur au moins 100 kilomètres de Meuse sur des périodes modélisées, cela donne un taux de croissance des rotifères proche de 0.55 jour $^{-1}$ à $20^{\circ} \mathrm{C}$ et donc un taux d'accroissement du peuplement de 0.3 jour $^{-1}$ à $20{ }^{\circ} \mathrm{C}$ (A20 $=0.3$ jour ' $)$, si l'on déduit les termes de disparition retenus par Everbecq et al. (0.125 jour ${ }^{-1}$ en moyenne pour la respiration et 0.125 jour ${ }^{\prime}$ en moyenne pour la mortalité).

II apparaît clairement qu'un tel niveau A20 $=0.3$ jour $^{1}$ surévaluerait la densité des rotifères en Loire moyenne, comme le montre la figure 7 dans laquelle sont comparées les densités mesurées avec les résultats que donnerait le modèle de la Loire présenté au chapitre 3.2 en choisissant A20 $=0.3$ jour $^{-1}$ sur l'ensemble de l'été. Pour un telle valeur de A20, on obtient en effet par le calcul un niveau moyen de concentration de rotifères sur l'été respectivement trois fois et six fois supérieur au niveau moyen des mesures effectuées à Muides et à Tours - alors que cet écart calcul-mesure n'est que de respectivement $2 \%$ et $10 \%$ à Muides et à Tours avec les valeurs de A20 calées au chapitre 3.2.

\section{ANALYSE DES RÉSULTATS ET CONCLUSION}

\section{On a donc vu :}

- que l'exploitation des mesures de densité de rotifères effectuées dans l'écoulement entre l'amont du confluent de l'Allier et Tours au cours de l'été 1982 donnait - par modélisation - un taux d'accrois- 
sement moyen du peuplement de 0.12 jour $^{-1}$ à $20^{\circ} \mathrm{C}$ (soit 0.2 jour $^{-1}$ à $25^{\circ} \mathrm{C}$ ) sur ce tronçon de Loire, avec un plafond de 0.22 jour $^{-1}$ pendant deux semaines au mois d'août. Ces chiffres résultent d'un prise en compte par excès des effets diluants induits par les apports de débit sur le parcours (Allier et apports aval, notamment nappe de Beauce et Loiret). Leur oubli aurait conduit à un taux d'accroissement moyen proche de 0 , puisque la valeur moyenne des densités de rotifères mesurées à Tours n'est supérieure que d'à peine plus de $5 \%$ à celle de la Loire dans le proche amont du confluent de l'Allier.

- que ce taux d'accroissement amont-aval donné par le modèle était nettement plus faible, d'une part que celui trouvé in vitro pour une durée d'incubation comparable au temps de transport des masses d'eau sur le tronçon de Loire étudié ; d'autre part de ceux habituellement considérés dans la bibliographie.

Comment expliquer un tel écart terrain/expérimentations?

Etant donné que le taux d'accroissement d'un peuplement est la différence entre son taux de croissance et son taux de disparition, une des deux hypothèses suivantes (ou un mixage des deux) apparaît possible :

- hypothèse 1 : les expérimentations in vitro sous-estiment le taux de disparition des rotifères dans le fleuve
- hypothèse 2 : les expérimentations in vitro surestiment le taux de croissance des rotifères dans le fleuve.

Explorons l'hypothèse 1. II s'agit donc de se demander pourquoi le taux de disparition des rotifères en rivière serait plus grand qu'in vitro.

Une première explication possible est que la mortalité des rotifères par prédation est moins forte dans les flacons de culture :

- il est vrai que les alevins de poissons consommateurs connus des rotifères n'y sont évidemment pas présents. La question alors posée est la capacité des alevins de poissons (dont la production annuelle en rivière est de l'ordre de $80 \mathrm{~kg}$ de poissons vifs/ha/an) à contrôler en été le développement des rotifères de Loire au point de générer chez ceux-ci un taux de disparition important. Point à considérer pour le creusement de cette hypothèse, il avait été établi en 1982 que les poissons les plus abondants en Loire (poison chat, goujon, hotus, chevesne, brême et gardon) se reproduisaient en mai - début juin, l'impact commençant 3 semaines après la ponte et durant près de 2 mois. Cette information est éventuellement à mettre en rapport avec la constatation que le taux d'accroissement du peuplement des rotifères est un plus élevé après le 10 août, à un moment où la pression de prédation par les alevins doit théoriquement commencer à diminuer. 
- en l'absence effective d'autres prédateurs zooplanctoniques, le gros rotifère Asplanchna est également connu comme l'autre consommateur principal possible du peuplement de rotifères en Loire. II peut représenter quelques pour cents du peuplement de rotifères sur la base des donnés disponibles pour les années antérieures à 1998 (Champ, 1980; Pourriot, 1982; Lair et al. 1997, Lair et al. 1998, Lair et al. 1999). La question alors posée est d'une part la capacité de ces gros rotifères à générer seuls ou en association avec les alevins de poissons un taux de disparition de l'ordre de 0.2-0.3 jour' pour l'ensemble du peuplement, d'autre part la possibilité d'une inhibition de leur développement dans les conditions de culture in vitro retenues.

Une deuxième explication possible est que le taux de disparition des rotifères par sédimentation est élevé en Loire. Compte tenu de la vitesse élevée de l'écoulement principal, cette sédimentation se produirait essentiellement au contact des îles et des berges.

Une troisième explication théoriquement possible pour l'hypothèse 1 serait que les conditions in vitro induisent une sélectivité en favorisant l'inhibition d'organismes ou de germes divers qui en rivière, ont pour effet d'augmenter le terme global de disparition naturelle (c'est à dire hors prédation) des rotifères. Un point à considérer pour soutenir éventuellement cette explication: la mortalité naturelle sur la durée des incubations in vitro présentées au chapitre 3.3 était négligeable, les rotifères trouvés à la fin apparaissant tous vivants.

Explorons maintenant l'hypothèse 2. Il s'agit de se demander pourquoi le taux de croissance des rotifères en Loire serait plus faible en rivière qu'in vitro.

Plusieurs sous-questions doivent alors être examinées :

- la croissance des rotifères peut elle être limitée par une présence insuffisante du stade trophique inférieur? Et si oui, pourquoi cette limitation serait moins importante in vitro? Considérons le phytoplancton, nourriture de base mais non exclusive pour les rotifères : il a eu sur l'été 1982 une teneur toujours comprise entre 140 et $250 \mathrm{mg}$ chlorophylle $a / m^{3}$ entre Bonny et Tours, et la majorité du temps entre 150 et $200 \mathrm{mg}$ chlorophylle $\mathrm{a} / \mathrm{m}^{3}$. Cela constitue en théorie une source d'alimentation très supérieure aux besoins des rotifères. Certes, il faut tenir compte du fait que la prédation des rotifères s'exerce avant tout sur les organismes de petite taille : Pourriot (1982) indique une taille maximale de particules ingérées de 20 micromètres pour Brachionus calyciflorus espèce dominante dans la Loire, la taille optimale de consommation variant selon lui entre 4 et 10 micromètres. Les analyses de spectre de taille effectuées au cours de la campagne d'été 1982 sur des diatomées - qui constituent le groupe dominant de phytoplancton en 
Loire moyenne avec les chlorophycées (Lair et al. 1997, Lair et al. 1998, Lair et al. 1999) - indiquent qu'à Tours, $85 \%$ des diatomées avaient une taille inférieure à 10 microns le 28 juillet pendant la période de stagnation voire même de diminution du peuplement de rotifères, qui avait alors une densité de l'ordre de $0.4^{*} 10^{6}$ individus $/ \mathrm{m}^{3}$. Ce pourcentage était à l'inverse de seulement $1 \%$ le 26 août en cette station, au moment où la densité de rotifères avait fortement augmenté les jours précédents et atteignait $1^{\star} 10^{6}$ individus $/ \mathrm{m}^{3}$. Comme ce niveau élevé de densité de rotifères n'a été observé que quelques jours et que les rotifères ont d'autres sources d'alimentation (autres algues, bactéries, détritus organiques), une limitation de la croissance des rotifères par manque de nourriture semble exclue et ne peut donc être mise en avant pour expliquer des différences avec les observations in vitro.

- les caractéristiques hydrauliques de la Loire moyenne ne freinent elles pas considérablement la croissance des rotifères? Lorsque l'hypothèse du rôle inhibiteur des vitesse élevées en Loire sur la croissance des rotifères a été récemment discutée avec N. Lair, celle-ci nous a fait remarquer qu'à se connaissance, personne n'avait réussi à étudier de façon entièrement convaincante in situ la filtration des rotifères en écoulement fluvial, les expérimentations utilisant des chambres ne reprodui- sant pas les conditions réelles d'écoulement (expérimentations de Gosselain (1998) par exemple). L'effet défavorable de la vitesse sur la croissance des rotifères était déjà suspectée par Pourriot en 1982 ; il est également suggéré par des auteurs ayant travaillé dans le cadre du programme PIREN Seine qui parlent de l'effet pénalisant d'une turbulence élevée (Boet et al., 1998). Pour creuser cette hypothèse, il faudrait s'intéresser probablement aux échelles de turbulence, car après tout, dans les incubations in vitro, une agitation de l'eau était maintenue pour empêcher la sédimentation des algues. Un point à considérer pour appuyer l'hypothèse du rôle inhibiteur de la vitesse du fleuve: Nicole Lair et Virginie Picard ont très récemment montré expérimentalement que la croissance de rotifères prélevés en Loire en amont de Blois était moindre dans un milieu artificiel maintenu en vitesse que dans un milieu stagnant (communication personnelle, article à paraître).

- enfin, question du même ordre posée pour le terme disparition, les conditions in vitro ne peuvent elles induire une sélectivité, en freinant le développement d'organismes ou de germes divers qui, dans le fleuve, auraient pour effet de limiter la croissance globale des rotifères?

En conclusion, le rôle de plusieurs facteurs reste à clarifier pour préciser l'importance relative de chacune des 
hypothèses 1 et $2:$ prédation par les Asplanchna et les alevins de poissons, sédimentation, vitesse/turbulence, effet sélectif des conditions in vitro pour le développement d'espèces de rotifères ou de germes pathogènes. On peut penser qu'une détermination spécifique des rotifères aurait permis d'en dire plus, notamment par comparaison de la densité des espèces dominantes de rotifères et d'Asplanchna le long du fleuve et au cours des incubations.

II est à noter que le seul choix de l'hypothèse 2 (c'est à dire sans considération de facteurs de l'hypothèse 1), qui consisterait à prendre des taux de croissance et de disparition très faibles, nécessite de supposer qu'une proportion non négligeable de rotifères passant au confluent Loire/Allier se retrouve à Tours, ce qui est théoriquement plausible: d'après $N$. Lair (communication personnelle), les rotifères sont physiologiquement tout à fait capables de se maintenir en survie au delà de la semaine dans un écosystème qui ne présenterait pas des conditions favorables pour la croissance.

Le seul choix de l'hypothèse 1 qui consisterait à prendre pour le fleuve les taux élevés de croissance trouvés in vitro - exige de retenir des taux de disparition également élevés, du fait que leur différence (A20) est 0.12 jour : en moyenne à $20^{\circ} \mathrm{C}$. La question alors posée est la possibilité que le système puisse rester durablement proche de l'équilibre - c'est à dire sans écart trop important de ces deux taux qui générerait des varia- tions amont-aval excessives de densités de rotifères?

On répondra en remarquant, en faveur de cette hypothèse 1 :

- que A20 n'est pas constant et peut varier entre 0.05 jour $^{-1}$ et 0.22 jour $^{-1}$ selon la période de l'été; la notion d'équilibre entre taux de croissance et taux de disparition apparaît ainsi toute relative;

- que des organismes comme les bactéries (N. Lair et $\mathrm{V}$. Picard, communication personnelle, articles à paraître) ou le phytoplancton (Lair et al. 1997, 1998, 1999, 2000) - qui ont physiologiquement des taux de croissance bien supérieurs aux rotifères - ont comme ces derniers, une gamme de variation de concentration relativement réduite en général (hors crues) en été le long de la Loire moyenne (en schématisant la plupart des mesures ne varient pas plus d'un facteur 2). Comme si l'édifice trophique marqué par une grande diversité biologique - qui conduit $\mathrm{N}$. Lair à considérer que cette partie du fleuve "se porte bien" (communication aux journées Hydroécologie de 2001) - avait une capacité d'autorégulation remarquable. On peut en particulier trouver étonnant que le niveau moyen de densités de rotifères au cours de l'été 1982 donné par les mesures soit en chaque station de Loire étudiée - depuis l'amont du confluent de l'Allier jusqu'à Tours compris entre $0.4^{*} 10^{6}$ individus $/ \mathrm{m}^{3}$ et $0.5^{*} 10^{6}$ individus $/ \mathrm{m}^{3}$, comme si 
l'effet diluant des affluents successifs sur le parcours était sans cesse compensé.

En ce qui concerne la mise au point de modèles de rotifères, les résultats obtenus suggèrent :

- que l'approche très simplifiée retenue dans le modèle de Gosse de 1981 consistant à supposer que la chronique de densité de rotifères disponible à Saint Laurent s'appliquait sur l'ensemble du tronçon de Loire moyenne modélisé n'aurait pas donné de trop mauvais résultats pour l'été 1982, compte de la faible augmentation amont-aval donnée par les mesures

- que l'approche consistant à appliquer directement sur le tronçon de Loire étudié le modèle récent publié par Everbecq et al. (2000) n'aurait pas été satisfaisante, celui-ci donnant des taux d'accroissement du peuplement trop grands.

On perçoit ainsi une fois encore l'importance de la collecte de mesures in situ sur la rivière dont on veut modéliser l'écosystème, compte tenu des risques potentiels d'une simple extrapolation d'expérimentations in vitro ou d'une application sans discussions de modèles établis sur une rivière différente.

\section{REMERCIEMENTS}

Michel KHALANSKI est vivement remercié pour son co-pilotage de la campagne de mesures présentée et ses informations transmises lors d'échanges fréquents sur l'analyse des résultats obtenus. Sont également remerciés : la sociéte AQUASCOPE, qui a assuré sous la responsabilité de Danièle LEVET les prélèvements d'eau aux 11 stations du réseau de mesure; Jean Michel PELATA qui a effectué les comptages de zooplancton et participé en collaboration avec Michel KHALANSKI et l'auteur à une première analyse des résultats dans le cadre d'un DEA d'Ecologie générale à l'Ecole Normale supérieure en 1983; Nicole LAIR pour sa réactivité aux questions posées sur le comportement des rotiferes lors de plusieurs échanges au cours de l'année 2000; et Jean Pierre DESCY de I'Université de Namur qui a apporté les éclaircissements demandés en 2000 sur les travaux entrepris par lui et ses collaborateurs sur le plancton fluvial.

\section{RÉFÉRENCES BIBIIOGRAPHIQUES}

Allan J.D, 1976 : Life history patterns in zooplankton; The american naturalist, vol 110, n⿳971 (165-180).

Boet P., Akopian M., Beiliard J., Berrebi R., Pourriot R., Tales E., Testard P., 1998 : Une faune aquatique sous pressions multiples. In La Seine et son bas$\sin$. Fonctionnement écologique d'un système fluvial anthropisé. Meybeck, de Marsily, Fustec Eds. Elsevier.

Champ P., 1977 : Etude des populations d'un rotifère épiphyte dans la Loire. Cahiers du laboratoire d'Hydrobiologie de Montereau. N4.

Champ P., 1980 : Biomasse et production du phytoplancton de la Loire en amont et en aval de la centrale nucléaire de Saint-Laurent des Eaux. Electricité de France. Rapport EDF HE-31-80-01, Paris, $32 p$.

Crouzet Ph., Receveur R., 1977 : Alimentation de la Loire moyenne par la nappe des calcaires de Beauce. Rela- 
tions avec la qualité des eaux. L'EAU EN Loire Bretagne, 14, 8-13.

Everbecq E., Gosselain V., Viroux L., Descy J.P., 2000 : POTAMON: A dynamic model for predicting phytoplankton composition and biomass in lowland rivers. Water research.

Gonzalez R., 1992 : Etude de l'organisation et évaluation des échanges entre la Loire moyenne et l'aquifère des calcaires de Beauce. Thèse Université d'Orléans. Orléans. Géosciences. Mémoire $n^{\prime} 8$.

Gosse Ph., 1981: Water quality Modelling in the middle reaches of the Loire river. In Mitsch W.J., BOSSERMAN R.W., KLOPATEK J.M. (Eds), Energy and Ecological Modeling, 281-291, Elservier, Amsterdam.

Gosselain V., 1998 : Phytoplancton de la Meuse et de la Moselle et Impact du broutage par le zooplancton. These. Facultés universitaires Notre Dame de la Paix de Namur. Presses Universitaires de Namur.

Khalanski M., Neau J., 1981 : Correction de l'équation trichromatique du SCOUNESCO pour la mesure de la chirophylle a non dégradée dans le plancton d'eau douce.. Electricité de France. Rapport EDF HE-31-81-45, Paris, $16 \mathrm{p}$.

Lair N. et Sargos D., 1981: Dynamique des populations planctoniques en Loire moyenne. Annales de la Station Biologique de Besse-en-Chandesse. 15, 60-?

Lair N., Reyes-Marchant P. 1997: The potamoplankton of the Middle Loire and the role of the moving littoral in downstream transfer of algae and rotifers. Hydrobiologia 356 : 33-52.

Lair N., Reyes-Marchant P.. Jacquet V. 1998 : Développement du phytoplancton, des Ciliés et des Rotifères sur deux sites de la Loire moyenne (France), en période d'étiage. Annls Limnol. 34(1) : 35-48.
Lair N., Jacquet V. and Reyes-Marchant P., 1999: Factors related to autotrophic potamoplankton, heterotrophic protists and micrometazoan abundance, at two sites in a lowland temperate river during low water flow. Hydrobiologia 394 : 13-28.

Lair N., Reyes-Marchant P. 2000 : Caractéristiques hydroécologiques de la Loire moyenne : bilan des études réalisées de 1977 a 1998 sur le site élecronucléaire de Saint Laurent des Eaux (France). Hydroécologie appliquée (ce numéro).

Liu Fennggi, 1996. Production and applications of rotifers in aquaculture. Aquaculture Magazine., 22/3, pp. 16-22.

Pourriot R., 1982 : Ecologie du plancton des eaux continentales. Ecologie. Masson (198 pages).

Pourriot R., Champ P., 1982 : Influence de quelques facteurs du milieu sur la structure et la dynamique saisonniere du zooplancton. Rapport EDF HE/3182.25. $54 \mathrm{p}$.

Pourriot R., Champ P., Tassigny M., 1982: Phytoplancton vernal de la Loire. Influence de quelques facteurs du milieu sur la structure et la dynamique saisonnière du zooplancton. Cahiers du laboratoire d'Hydrobiologie de Montereau. N"14;35-44.

Pourriot R., Benest D., Champ P., Rougier C., 1982 : Infleunce de quelques facteurs du milieu sur la composition ef la dynamique saisonnière du zooplancton de la Loire. Acta oecol., oecol. Gener. 3, 353-371.

Siclet F., Gailhard J., Ciffroy P., 2001: Radionuclides concentrations in the Loire river system resulting from routine discharges of five nuclear power plants. Article accepté au congrès IPSN ECORAD 2001 (3-8 septembre 2001).

Viroux L., 2000 : Dynamique du métazooplancton en milieu fluvial. These. Facultés universitaires Notre Dame de la Paix de Namur. Namur. Belgique. 